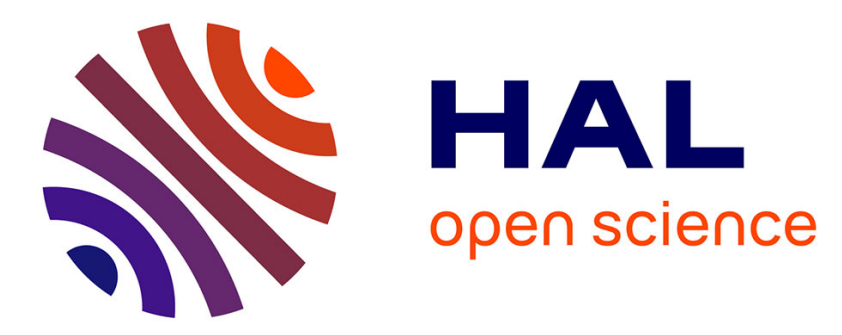

\title{
On-line spectroscopic study of brominated flame retardant extraction in supercritical $\mathrm{CO} \_2_{2}$
}

Dong Xia, Ange Maurice, Antoine Leybros, Jong-Min Lee, Agnès Grandjean, Jean-Christophe Gabriel

\section{- To cite this version:}

Dong Xia, Ange Maurice, Antoine Leybros, Jong-Min Lee, Agnès Grandjean, et al.. On-line spectroscopic study of brominated flame retardant extraction in supercritical CO_2. Chemosphere, 2020, 263, pp.128282. 10.1016/j.chemosphere.2020.128282 . cea-02936108

\section{HAL Id: cea-02936108 https://hal-cea.archives-ouvertes.fr/cea-02936108}

Submitted on 11 Sep 2020

HAL is a multi-disciplinary open access archive for the deposit and dissemination of scientific research documents, whether they are published or not. The documents may come from teaching and research institutions in France or abroad, or from public or private research centers.
L'archive ouverte pluridisciplinaire $\mathbf{H A L}$, est destinée au dépôt et à la diffusion de documents scientifiques de niveau recherche, publiés ou non, émanant des établissements d'enseignement et de recherche français ou étrangers, des laboratoires publics ou privés. 


\section{On-Line Study of Flame Retardants Extraction in Supercritical $\mathrm{CO}_{2}$}

2 Dong Xia ${ }^{\mathrm{a}}$, Ange Maurice ${ }^{\mathrm{a}}$, Antoine Leybros ${ }^{\mathrm{b}}$, Jong-Min Lee ${ }^{\mathrm{a}, \mathrm{c}}$, Agnes Grandjean $^{\mathrm{b}}$ and Jean-Christophe P.

3 Gabriel ${ }^{\mathrm{a}, \mathrm{d}^{*}}$

$4 \quad{ }^{a}$ Nanyang Technological University, Energy Research Institute @ NTU (ERI@N), SCARCE Laboratory,

5 637459, Singapore.

6

${ }^{\mathrm{b}}$ CEA, DES, ISEC, DMRC, Univ Montpellier, Marcoule, France

7

${ }^{\mathrm{c}}$ Nanyang Technological University, School of Chemical and Biomedical Engineering, 637459, Singapore.

8

d Université Paris-Saclay, CEA, CNRS, NIMBE, 91191, Gif-sur-Yvette, France.

$9 \quad{ }^{*}$ Corresponding author. Jean-Christophe P. Gabriel

E-mail address: Jean-christophe.gabriel@cea.fr / jgabriel@ntu.edu.sg

\section{ABSTRACT}

Extraction of brominated flame retardants (BFRs) from polymers before disposal or recycling will alleviate negative environmental effects and ensure safe usage of recycled products. Supercritical $\mathrm{CO}_{2}$ is appealing as solvent owing to its green properties but also challenging due to its limited solvation power towards polar molecules. For a more comprehensive evaluation of supercritical extraction potentialities, we (i) developed an on-line analytical technique compatible with both UV-vis and FTIR spectroscopies to enable kinetic and thermodynamic studies; (ii) studied kinetic extraction of three conventional and two novel BFRs. Concentration at saturation were determined by gravimetric method or X-ray fluorescence. When compared to UV-vis, FTIR exhibited a higher applicability to follow BFR extraction's time-dependency binary and ternary systems. Faster stirring speed, higher temperature, and finer particle size were found to accelerate overall extraction kinetics. In 
binary systems, times required to achieve equilibrium for each BFR were less than 2 hours at $60{ }^{\circ} \mathrm{C}, 25 \mathrm{MPa}$ and for their low solubility if one uses flowing supercritical $\mathrm{CO}_{2}$.

Keywords: Extraction; Kinetic; Brominated Flame Retardant; Spectroscopy; Supercritical $\mathrm{CO}_{2}$; On-line analysis

\section{Introduction} amount of polymeric materials into people's daily life (Alaee, 2003). To ensure safety and compliance with flammability standards, the use of flame retardants (FRs) in various polymeric products is essential. Among all kinds of FRs, brominated flame retardants (BFRs) are the most effective because they require the lowest amount for the highest flame retardancy, thus their global market demand continues to increase substantially (Birnbaum and Staskal, 2004; Bergman et al., 2012; Gramatica et al., 2016). At present, the market is witnessing a gradual shift in demand for novel BFRs, in part driven by the phasing-out of widely used BFRs such as polybrominated diphenyl ethers, now regulated by Stockholm Convention due to concerns over risks to public and ecosystem health (Bramwell et al., 2017; Stubbings et al., 2019). Apart from more than seventy-five kinds of conventional BFRs, around sixty kinds of novel BFRs were reported to be used world-wide but also be ubiquitous in the environment (Georlette, 2001; Liu et al., 2016; Yu et al., 2016; Zuiderveen et al., 2020). Detailed reviews on 

as hormone disruption and genotoxicity (Kim et al., 2014; Xiong et al., 2019). Incineration has the disadvantage

of generating corrosive or gases and possibly highly toxic dioxins (Sakai et al., 2001; Ni et al., 2012).

Landfilling results in the leaching of toxic brominated substance with the possible transfer into local aquifers, especially in the case of additive BFRs that are simply blended with polymers (Zhou et al., 2013; Cristale et al., 2019). Even in mechanically recycled plastics, uncontrolled BFR additives can cause fluctuation in their quality.

Therefore, the removal of BFRs from polymers has been a necessity in order to recycle or even simply dispose them.

Solvent-based extraction has been proposed to remove BFRs from polymeric substrates with organic solvents, such as toluene, methanol, or glycol ether based compounds (Nakajima et al., 2002; Altwaiq et al., most of their original qualities. Furthermore, solvents could be reused by distillation. Hence, some commercial processes have been reported, like CreaSolv, which uses a combination of solvents to extract various BFRs the related health and safety issues (toxicity, flammability, risks associated with distillation). Another drawback of the traditional solvent extraction is that the subsequent distillation and drying processes are energy-intensive, leading to increase in operational costs.

BFRs, mainly because it is cheap, non-toxic and non-flammable by nature, has high mass and thermal 
(Taylor, 1996; Beckman, 2004). Solubility or saturated concentration is one of the most important parameters to

determine limits of extractability, thus, solubilities of some conventional BFRs have been measured in $\mathrm{ScCO}_{2}$ as function of pressure and temperature (Gamse et al., 2000; Peng et al., 2014). Also, $\mathrm{ScCO}_{2}$ extraction of BFRs was proved to be of various degrees of success in extraction efficiency with or without co-solvents, although the solvation power of $\mathrm{ScCO}_{2}$ is generally limited towards polar or high molecular weight compounds (Marioth et al., 1996; Suzuki et al., 2002; Altwaiq et al., 2003). Otherwise, extraction kinetics must be investigated to validate process scale-up and develop successful industrial processes (Nimet et al., 2011; Sodeifian et al., 2016; Villanueva-Bermejo et al., 2020). Despite this, to our knowledge, there have only been few such studies reported previously, either off-line or on-line, for BFR extraction (Bunte et al., 1996; Wang et al., 2004). This study proposes to evaluate feasibility of BFR extraction using $\mathrm{ScCO}_{2}$ considering thermodynamic and kinetic point of view. We therefore report here a method coupling versatile high pressure vessel with both on-line UV-vis and mid-IR spectrometers and demonstrate their applicability: (i) to perform spectroscopic analysis of multicomponent mixtures such binary and ternary mixtures in $\mathrm{ScCO}_{2}$ media; and (ii) to investigate effects of temperature, pressure, stirring, and matrix size on extraction kinetics for five BFRs, including three of the most used conventional ones and two popular novel ones.

\section{Materials and methods}

\subsection{Materials}

Five selected BFR compounds: (i) 3,3',5,5'-Tetrabromobisphenol A (TBBPA) and (ii) 1,2,5,6,9,10-

Hexabromocyclododecane (HBCD) were purchased from Sigma-Aldrich (St. Louis, MO, USA); (iii)

Decabromodiphenyl ether (DBDBE) and (iv) 2,4,6-Tris(2,4,6-tribromophenoxy)-1,3,5-triazine (TTBP-TAZ)

were purchased from Macklin (Shanghai, China); (v) 2,2-Bis[3,5-dibromo-4-(2,3- 
dibromopropoxy)phenyl]propane (TBBPA-BDBPE) was purchased from Aladdin (Shanghai, China). The BFRs'

Chemical structures and other detailed information are presented in Fig. S. 1 and table S. 1, respectively. Since $\mathrm{HBCD}$ and $\mathrm{DBDBE}$ have relative lower purities, they were purified in flowing $\mathrm{ScCO}_{2}$ at $60{ }^{\circ} \mathrm{C}$ and $25 \mathrm{MPa}$ for $6 \mathrm{~h}$ (Gamse et al., 2000). The other BFR compounds were used without any further purification.

$$
\text { Ultra-pure water was obtained from a water purification system (WaterPro }{ }^{\circledR} \text {, Labconco Co., USA), with a }
$$
resistivity of $18.2 \mathrm{M} \Omega \cdot \mathrm{cm}$ at $25^{\circ} \mathrm{C}$.

Liquid $\mathrm{CO}_{2}$ was supplied by CryoExpress Pte. Ltd. (Singapore) with a purity of $99.9 \%$.

Acrylonitrile butadiene styrene (ABS) resin was selected as typical polymer substrate for additive BFRs because it is commonly used in consumer products, such as housing of electric appliances, electronics, and toys. BFR-free ABS and BFR-containing ABS pellets were fabricated as industrial practices by Shandong Tianyi Chemical Co., Ltd. (China) as follows. Industrial grade TBBPA and TBBPA-BDBPE were respectively incorporated in the BFR-free ABS resin by hot blending with a proportion of $13 \mathrm{wt} . \%$ and the resulted mixtures were extruded to obtain pellets. We then grinded these as-purchased pellets into powders with a centrifugal mill (ZM 200, Retsch, Germany), at cryogenic temperature using liquid $\mathrm{N}_{2}$, then sieved the powders into two particle size fractions $\left(D_{\mathrm{ABS}}\right)$ in the ranges: (i) $0.25-1 \mathrm{~mm}$, and (ii) less than $0.25 \mathrm{~mm}$.

\subsection{Experimental apparatus}

A schematic representation of our $\mathrm{ScCO}_{2}$ extraction system optically coupled with on-line spectrometers (FTIR and UV-vis) is shown in Fig. 1. The core part of the system, the extraction reactor, is a commercial pressure vessel from Parr Instrumental Company, USA (ref. 2430HC2), modified as per our requirements with optical windows to enable spectroscopic studies for operating pressure and temperature ranging up to $35 \mathrm{MPa}$ and $200{ }^{\circ} \mathrm{C}$. The vessel has an entire volume of $75.4 \mathrm{~mL}$, with minimized dead volumes from filling and venting ports. Two dismountable windows (made of sapphire for FTIR or fused silica for UV-vis; rupture modulus: 1200 
MPa versus $100 \mathrm{MPa}$; diameter: $25 \mathrm{~mm}$; thickness: $15.7 \mathrm{~mm}$ ) are located on each side of the vessel in opposition to each other. Each window is maintained in place thanks to a window holder and sealed by a Teflon O-ring and a Teflon cushion through "opposed force" (Fig. S. 2). The resulted optical path length is measured of $58 \mathrm{~mm}$, sufficiently long to give desired sensitivity and limits of detectability (Jackson et al., 1995; Poliakoff et al., 1995).

For the other parts of the $\mathrm{ScCO}_{2}$ extraction system (Fig. 1a), liquid $\mathrm{CO}_{2}$ coming from a liquid tank is first cooled by a circulation chiller (F250, Julabo, Germany) prior to its delivery to a pressure dual piston pump (LDClass, Teledyne ISCO, USA). The vessel temperature is monitored using a K-type thermocouple inserted into a stainless steel plunger. Two PID controlled (E5CC, Omron, Japan) electric cartridge heaters, slid into cavities in the bottom of the vessel, are used to raise the vessel's temperature. A magnetic agitator (MR $3001 \mathrm{~K}$, Heidolph, Germany) drives a $12 \times 3 \mathrm{~mm}$ Teflon-coated stirring bar to stir the contents in the vessel. Additional accessories permit measurements of pressure, temperature, and agitation speed with a precision of $0.1 \mathrm{MPa}, 0.1^{\circ} \mathrm{C}$, and $3 \%$, respectively.

(a)

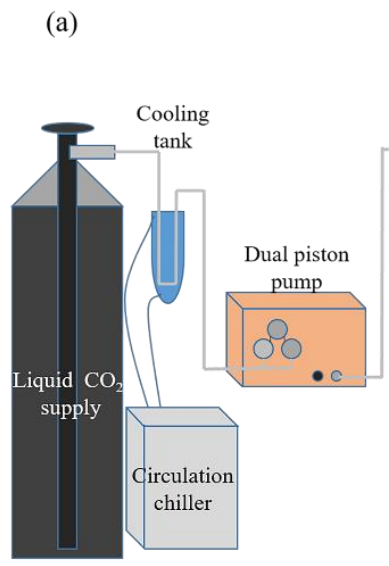

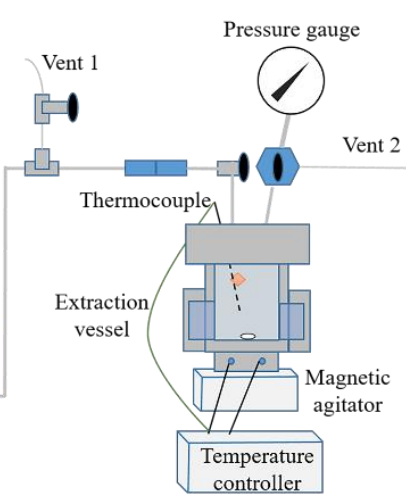

controller

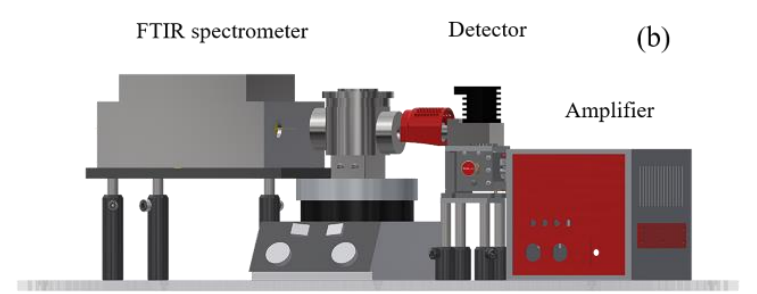

(c)

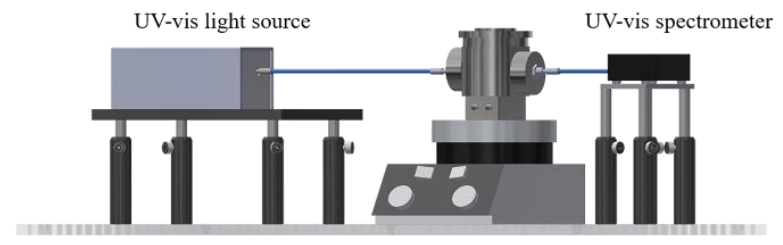

Fig. 1. Schematic illustration of the experimental $\mathrm{ScCO}_{2}$ extraction system (a), where each component of the system is not presented in proportion; the virtual deployment of the vessel with FTIR (b) and with UV-vis spectroscopic components (c), and the vessel head parts (model 4792) and the spilt rings (A455HC) are abridged for clearly presenting their optical 
alignment.

The spectroscopic components are combined with the $\mathrm{ScCO}_{2}$ extraction system in a modular fashion (Fig.

$1 \mathrm{~b}$ and Fig. 1c). Their optical alignment is readily adjusted thanks to bases and supporting rods purchased from Thorlabs Inc. (USA). The IR beam provided by a FTIR spectrometer (Bruker Alpha OEM, Bruker Optics Inc., Germany) transmits through the vessel, and the signal are detected by a Stirling Cycle Cooled detector connected with an amplifier (K508-MCT1000, Infrared Associates, U.S.A). They were initially integrated as an on-line monitoring of microfluidic processes (C. Penisson, 2018; Kokoric et al., 2018; Maurice et al., 2020) and have been reconverted here for the purpose of its extraction reactor.

For UV-vis monitoring, the UV radiation emanating from a deuterium light source (DH-MINI, Ocean

Optics Inc., UK) transfers through the vessel, a pair of optical fibers, and several collimating lens. Spectra were recorded by a UV-vis spectrometer (Flame-T, Ocean Optics Inc., UK).

\subsection{Measurement of extraction kinetics}

All BFR extraction experiments in $\mathrm{ScCO}_{2}$ were carried out using a solid-supercritical fluid extraction process. Prior to each extraction, a thorough clean-up of the $\mathrm{ScCO}_{2}$ extraction system was performed with flowing $\mathrm{ScCO}_{2}$ until no extra absorbance of contents was detected, and then reference spectra of pure $\mathrm{ScCO}_{2}$ were taken using identical operating conditions as sample spectra. To assess reproducibility and improve precision, each extraction was performed in triplicate. Precisely weighted pure BFR powders (100 mg) or ABS powders $(600 \mathrm{mg})$ were enclosed within Solidweave mesh (Mesh size $=9 \mu \mathrm{m})$ folded uniformly like a mailing envelope, which was then attached inside the vessel at the top of the thermocouple inverted finger, close to the filling port. After loading the sample, the vessel was hermetically closed and then flushed three times with gaseous $\mathrm{CO}_{2}$. Then it typically took around 10 minutes to heat it and fill it in with compressed $\mathrm{CO}_{2}$ to the desired temperature and pressure, prior to stirring at a set speed. Spectra were collected at regular intervals 
during the whole extraction process. IR spectra were collected with a $4 \mathrm{~cm}^{-1}$ resolution and were obtained by the

147

Fourier transformation of 64 interferograms. UV spectra were averaged from 1000 scans within an integration time of $0.015 \mathrm{~s}$. Each extraction experiment was terminated after reaching a steady state where spectra no longer show any change in absorbance. The decompression procedure was fast (less than $10 \mathrm{~s}$ ) along with subsequent refilling with gaseous $\mathrm{CO}_{2}$ and no re-deposition of solute back to sample mesh could be observed under a microscope $(\times 500$, RS Pro).

\subsection{Determination of saturated concentration}

Saturated concentration $\left(c_{\mathrm{s}}\right.$, in $\left.\mathrm{mmol} / \mathrm{L}\right)$ is the maximum concentration of a solute dissolved in a solvent. $c_{\mathrm{s}}$ of individual pure BFR in $\mathrm{ScCO}_{2}$ was measured gravimetrically using an analytical balance (MS105, MettlerToledo, USA) with a readability of $0.01 \mathrm{mg}$ and was calculated with the following equation:

$c_{\mathrm{s}}=10^{3} \cdot\left(m_{2}-m_{1}\right) /\left[M_{\mathrm{BFR}} \cdot\left(V_{\mathrm{v}}-V_{s}\right)\right]$

where $m_{1}$ and $m_{2}$ indicate the weight of sample package before and after extraction; $M_{\mathrm{BFR}}$ denotes the molar mass of the used BFR molecule. $V_{\mathrm{v}}$ is the vessel volume $(75.4 \mathrm{~mL})$ and $V_{\mathrm{s}}$ is the sample package volume determined with the water discharging method.

Since mass removal of BFR is not measurable gravimetrically for ABS samples, $\mathrm{Br}$ content changes of ABS samples before and after extraction were determined with an X-ray fluorescence analyzer (XRF) (Vanta C, Olympus, Japan) applying RoHS plus method. For this determination, the powdered ABS samples (200 mg) were pressed into a circular shaped disc using a hydraulic crimper (MSK-510M, MTI Co., China) at $160{ }^{\circ} \mathrm{C}$ and $5 \mathrm{MPa}$ for $5 \mathrm{~min}$. The mass removal of BFR can be calculated by dividing Br content of BFR from Br content difference of the ABS sample, thus the saturated concentration can be calculated with Eq. (1) as well. All experimental data was displayed in a format of mean value \pm standard deviation. 


\subsection{IR spectroscopy and data processing}

The IR spectra were processed with a software combination of OPUS 7.5 and Python 3.6.8. After baseline correction, the corresponding absorbance peak of interest (A) (Table S. 2) was integrated in the wavenumber region of $w_{2}-w_{1}\left(\right.$ in $\left.\mathrm{cm}^{-1}\right)$ using the following equation:

$A=\int_{w_{1}}^{w_{2}} \log _{10}\left(\frac{I_{0}}{I_{\mathrm{t}}}\right) \mathrm{d} w$

with $I_{0}$ and $I$ as sample and baseline intensity, respectively.

According to Beer-Lambert Law, the integrated absorbance was proportionally converted to BFR concentration $\left(c\right.$, in mmol/L) in $\mathrm{ScCO}_{2}$ (IUPAC, 1997):

$c_{\mathrm{t}}=A_{\mathrm{t}} \cdot c_{\mathrm{s}} / A_{\mathrm{s}}$

where the subscripts $t$ and $\mathrm{s}$ indicate timely and saturation readings.

Furthermore, the linear sum of the absorbance spectra of each component $\left(A_{\mathrm{i}}\right)$ was considered for quantitative interpretation of the overlapped peak area of a mixture $\left(A_{\mathrm{m}}\right)$ :

$A_{\mathrm{m}}=\sum_{i=1}^{N} A_{\mathrm{i}}$

\subsection{Mathematical modelling of extraction kinetics}

In outline, the $\mathrm{ScCO}_{2}$ extraction of compounds from solid matrices (porous or not) can be condensed into a four-step process: (i) diffusion of $\mathrm{ScCO}_{2}$ into internal structure of solid matrices; (ii) desorption and solvation of solute in the $\mathrm{ScCO}_{2}$; (iii) intra-particle diffusion of the mixture of solute and $\mathrm{ScCO}_{2}$ to solid interface; and (iv) diffusion of the mixture from the solid interface to bulk $\mathrm{ScCO}_{2}$ (Madras et al., 1994; Sunarso and Ismadji, 2009; Huang et al., 2012). All these steps are diffusion limiting steps except for the solubilization step (ii).

To achieve specific description of extraction kinetics involving complex phenomena with mathematical models, we introduced two simplified scenarios, including absence of matrix and well stirred reactor, based on the assumption that the diffusion steps can be controlled by changing of matrix properties or stirring speed, and 
hence basic physicochemical models could be applied (Srinivasan et al., 1990). For the solubilization step, an irreversible and first order model (linear desorption) was applied to calculate solubilization rate coefficient $\left(k_{\mathrm{s}}\right.$, in $\mathrm{h}^{-1}$ ) over extraction period $(t$, in $\mathrm{h}$ ) with the following equation (Tan and Liou, 1989; Elektorowicz et al., 2007;

Jokić et al., 2010):

$\frac{d c}{d t}=k_{\mathrm{S}}\left(c_{\mathrm{s}}-c_{\mathrm{t}}\right)$

and with the initial condition:

$t=0 \mathrm{~h}, c_{\mathrm{t}}=0 \mathrm{mmol} / \mathrm{L}$

After solving by integration on both sides of the Eq. (5), one can get

$\ln \left[\left(c_{\mathrm{s}}-c_{\mathrm{t}}\right) / c_{\mathrm{s}}\right]=-k_{\mathrm{S}} \cdot t$

The applied model for the diffusion steps is based on the hot sphere diffusion model derived from Fick's diffusion laws assuming that loaded particles consist of solid spheres with uniform radius and initial concentration of extracted substance and are immersed into a fluid free from the substance (Crank, 1979; Bartle et al., 1990; Huang et al., 2012). The model is expressed as:

$\frac{c_{\mathrm{t}}}{c_{\mathrm{s}}}=1-\frac{6}{\pi^{2}} \sum_{n=1}^{\infty} \frac{1}{n^{2}} \cdot \exp \left[-\left(\frac{n \pi}{R}\right)^{2} D_{\mathrm{e}} t\right]$ with $D_{\mathrm{e}}$ as effective diffusion coefficient in a solid substrate $\left(\mathrm{m}^{2} / \mathrm{h}\right)$ and $\mathrm{R}$ as radius of spherical solid particles (m).

For long time, all terms except the first in the series of exponential terms in Eq. (8) become negligible and the kinetic curve can be rewritten as:

$\ln \left[\left(c_{\mathrm{s}}-c_{\mathrm{t}}\right) / c_{\mathrm{s}}\right]=\ln \left(\frac{6}{\pi^{2}}\right)-\frac{\pi^{2} D_{\mathrm{e}}}{R^{2}} \cdot t$

where $\pi^{2} D_{\mathrm{e}} / \mathrm{R}^{2}$ may also be recast as diffusion rate coefficient $k_{\mathrm{D}}\left(\mathrm{h}^{-1}\right)$.

From Eq. (7) and Eq. (9), a plot of $\ln \left[\left(c_{\mathrm{s}}-c_{\mathrm{t}}\right) / c_{\mathrm{s}}\right]$ versus $\mathrm{t}$ may fall onto two intersecting straight lines with different slops $k_{1}$ and $k_{2}$ (Subra et al., 1998; Hojnik et al., 2008). 
211 In addition, absolute average relative deviations $(A A R D, \%)$ between experimental data $\left(c_{\mathrm{t}, \mathrm{exp}}\right)$ and modeled data

212

213

214

215

216

$\left(c_{t, \text { mod }}\right)$ are also evaluated by:

$A A R D=\frac{100}{N} \sum_{i=1}^{N} \frac{\left|c_{\mathrm{t}, \exp }-c_{\mathrm{t}, \mathrm{mod}}\right|}{c_{\mathrm{t}, \mathrm{exp}}}$

\section{Results and discussion}

\subsection{Comparison between FTIR and UV-vis spectroscopies}

Spectroscopic monitoring, including vibrational and electronic spectroscopies, is a classical on-line technique in many supercritical process studies because it allows for rapid and inexpensive determination of concentration with relatively small amounts of compound (Laintz et al., 1991; Carrott and Wai, 1998; Wang et

\section{al., 2004; Wang et al., 2018). In this study, FTIR and UV-vis spectroscopies were first compared for their} applicability to characterize BFR compounds in $\mathrm{ScCO}_{2}$. Raman spectroscopy was not considered since it suffers from a lack of sensitivity when solute concentrations are low (Jackson et al., 1995). In terms of spectrum window, the FTIR reference spectrum was narrowed to two available bands of $4500-3880 \mathrm{~cm}^{-1}$ and of 3500 $2600 \mathrm{~cm}^{-1}$ (Fig. S. 3a). The other IR bands, including the characteristic C-Br group $\left(690-515 \mathrm{~cm}^{-1}\right)$, were completely obscured by absorptions of sapphire windows and $\mathrm{ScCO}_{2}$, itself (Poliakoff et al., 1995). To circumvent this problem, some identifiable groups, like $v(\mathrm{C}-\mathrm{H})$ and $v(\mathrm{O}-\mathrm{H})$, were employed to characterize BFRs in $\mathrm{ScCO}_{2}$ (Table S. 2). However, due to the ubiquity of these groups in organic chemicals, the high purity of BFR compounds and the cleanliness of reference spectra are essential to characterize $\mathrm{BFRs}$ in $\mathrm{ScCO}_{2}$, especially for a quantitative purpose. Comparatively, the spectrum window of UV-vis could extend to almost the whole spectrometer's range (200-800 nm) (Fig. S. 3b).

With well-defined reference spectra, TBBPA, the most commercially used BFR, was characterized as an example in $\mathrm{ScCO}_{2}$ using FTIR and UV-vis spectrometers, respectively (Fig. 2). Both spectra demonstrated 
232

233

234

incremental absorbance over extraction time until they leveled off and reached a stable value. The FTIR spectrum of TBBPA was marked by two bands, with two maximum absorbance located at $2904 \mathrm{~cm}^{-1}$ and 3000 $\mathrm{cm}^{-1}$, resulting from $v(\mathrm{C}-\mathrm{H}$ alkane $)$ and composite $v(\mathrm{C}-\mathrm{H}$ combinations) absorption, respectively (Fulton et al., 1993). In the UV-vis spectrum, TBBPA showed two absorption band (200-260 nm and 260-310 nm) as a result of electronic transitions, $\pi \rightarrow \pi^{*}$ transition (conjugated $\mathrm{C}=\mathrm{C}$ group), which was similar to the spectrum of TBBPA through organic solvent presented previously (Lu et al., 1999; Khaled et al., 2018). However, the UVvis spectrum of TBBPA exposed an absorbance saturation problem. The high absorptivity of the chromophore in the UV region set an upper limit of detection, although the issue can be modified by replacing thicker windows to shorten the optical path length. Besides, a shift of peaks in the UV-vis spectrum was apparent with the increase of concentration, which resulted in the ambiguous peaks' interpretation.
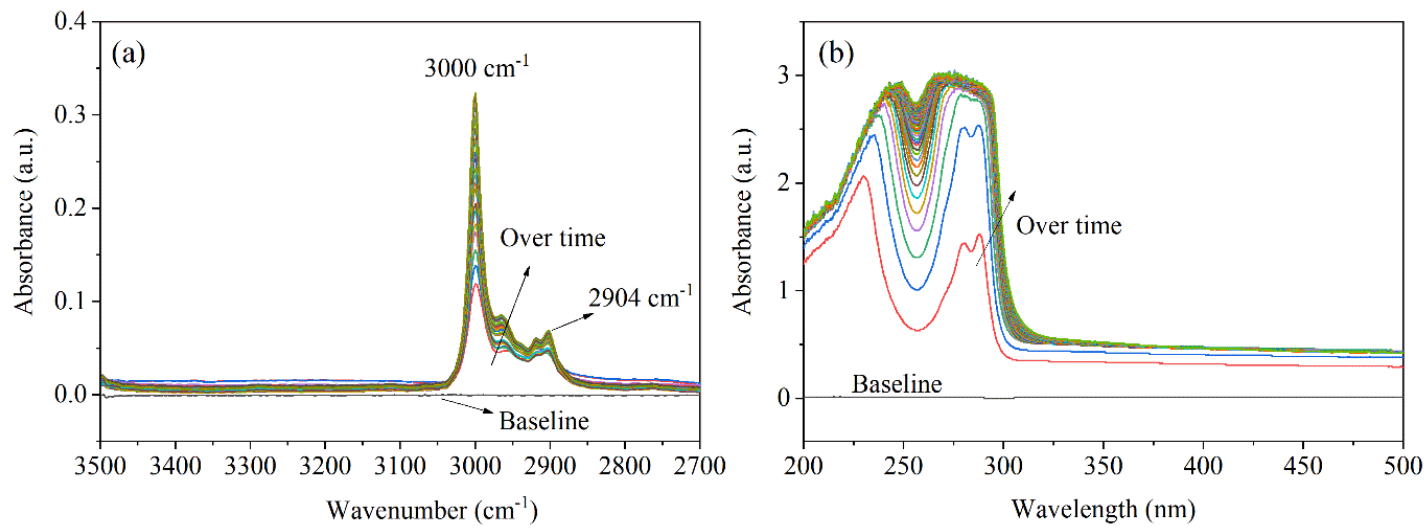

Fig. 2 FTIR (a) and UV-vis (b) spectra of TBBPA in $\mathrm{ScCO}_{2}$ over extraction period at $60^{\circ} \mathrm{C}, 20 \mathrm{MPa}$, and $1000 \mathrm{rpm}$.

Although each of the technique has its own advantages and shortcomings from the spectroscopic standpoint, FTIR is a better technique than UV-Vis for carrying out the on-line monitoring. In addition, the higher rupture modulus of sapphire than fused silica will facilitate a safer operating condition under high pressure. Therefore, only FTIR spectroscopy was used in the following sections. 

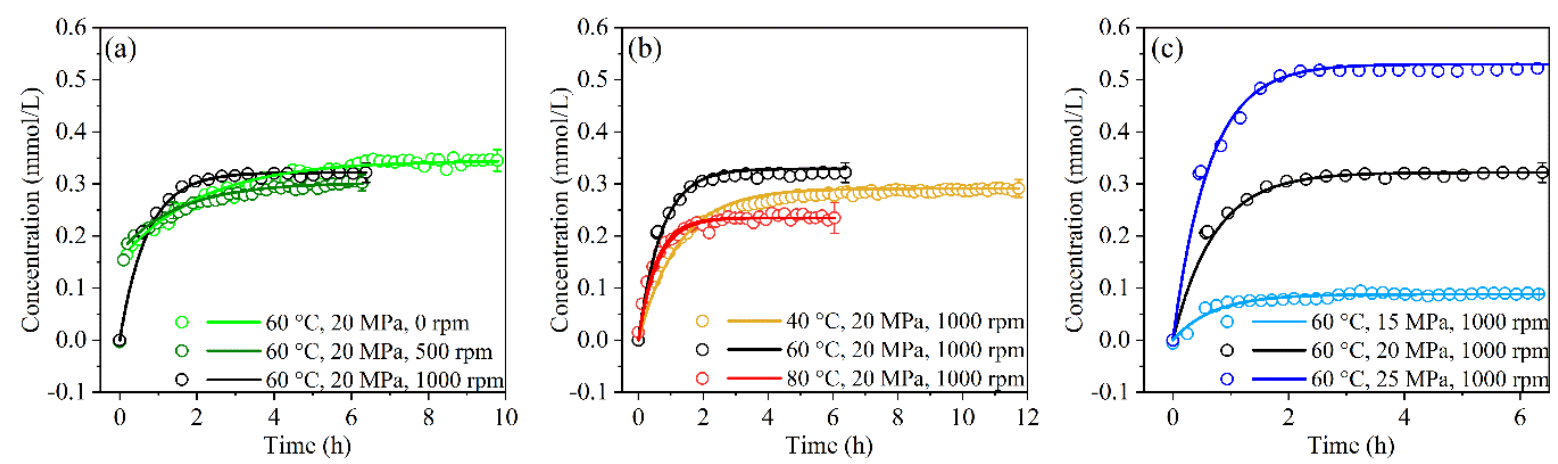

Fig. 3 Influence of operating conditions on $\mathrm{ScCO}_{2}$ extraction kinetics of TBBPA: stirring speed (a), temperature (b), and 


\begin{tabular}{|c|c|c|c|c|}
\hline BFRs & Operating conditions & $c_{\mathrm{s}}$ & $k \mathrm{~s}$ & $A A R D$ \\
\hline & & $\mathrm{mmol} / \mathrm{L}$ & $h^{-1}$ & $\%$ \\
\hline \multirow[t]{7}{*}{ TBBPA } & $40^{\circ} \mathrm{C}, 20 \mathrm{MPa}, 1000 \mathrm{rpm}$ & $0.291 \pm 0.004$ & $0.81 \pm 0.04$ & 3.16 \\
\hline & $60{ }^{\circ} \mathrm{C}, 15 \mathrm{MPa}, 1000 \mathrm{rpm}$ & $0.088 \pm 0.004$ & $1.43 \pm 0.19$ & 4.14 \\
\hline & $60^{\circ} \mathrm{C}, 20 \mathrm{MPa}, 0 \mathrm{rpm}$ & $0.345 \pm 0.021$ & $0.48 \pm 0.03$ & 2.52 \\
\hline & $60{ }^{\circ} \mathrm{C}, 20 \mathrm{MPa}, 500 \mathrm{rpm}$ & $0.302 \pm 0.016$ & $0.66 \pm 0.04$ & 2.28 \\
\hline & $60{ }^{\circ} \mathrm{C}, 20 \mathrm{MPa}, 1000 \mathrm{rpm}$ & $0.322 \pm 0.019$ & $1.40 \pm 0.04$ & 1.11 \\
\hline & $60{ }^{\circ} \mathrm{C}, 25 \mathrm{MPa}, 1000 \mathrm{rpm}$ & $0.531 \pm 0.014$ & $1.59 \pm 0.08$ & 2.22 \\
\hline & $80^{\circ} \mathrm{C}, 20 \mathrm{MPa}, 1000 \mathrm{rpm}$ & $0.235 \pm 0.007$ & $1.84 \pm 0.09$ & 3.70 \\
\hline HBCD & $60{ }^{\circ} \mathrm{C}, 25 \mathrm{MPa}, 1000 \mathrm{rpm}$ & $0.064 \pm 0.004$ & $4.68 \pm 1.09$ & 4.22 \\
\hline DBDPE & $60^{\circ} \mathrm{C}, 25 \mathrm{MPa}, 1000 \mathrm{rpm}$ & $0.004 \pm 0.000$ & N.C. & N.C. \\
\hline TBBPA-BDBPE & $60^{\circ} \mathrm{C}, 25 \mathrm{MPa}, 1000 \mathrm{rpm}$ & $0.154 \pm 0.002$ & $4.68 \pm 0.91$ & 5.70 \\
\hline TTBP-TAZ & $60{ }^{\circ} \mathrm{C}, 25 \mathrm{MPa}, 1000 \mathrm{rpm}$ & $0.012 \pm 0.000$ & $8.10 \pm 1.06$ & 3.71 \\
\hline
\end{tabular}

N.C. means not calculated.

Stirring was found to improve the extraction kinetic and shorten the time required to achieve saturation from 6 hours down to 2 hours, when comparing the three cases of non-stirring, $500 \mathrm{rpm}$, and $1000 \mathrm{rpm}$ (Fig. $3 \mathrm{a}$ ).

Only at the highest stirring speed could the overall experimental scatterplots be fitted well with a first order kinetic equation (Eq. 5), indicating the external diffusion step is negligible in the well stirred reactor (the second simplified scenario, see section 2.6) by fast stirring and leading to a solubilization-dominated process. Whereas, external diffusion becomes more significant with less powerful stirring. Since the rapid increase of concentration during the initial compression period was owing to the inevitable agitation caused by filling 
turbulences, the fitting model was applied after the initial compression period in the $0 \mathrm{rpm}$ and $500 \mathrm{rpm}$ cases, where the decreased $k_{\mathrm{S}}$ can only be attributed to external diffusion. Besides, stirring had insignificant impact on the solubility of TBBPA considering the measurement's uncertainties of the experiments.

The effects of temperature and pressure on extraction of TBBPA were explored at a constant stirring speed of $1000 \mathrm{rpm}$ (Fig. $3 \mathrm{~b}$ and Fig. $3 \mathrm{c}$ ). The $k_{\mathrm{S}}$ value more than doubled with the increase of temperature from $40{ }^{\circ} \mathrm{C}$ to $80{ }^{\circ} \mathrm{C}$, while the highest $c_{\mathrm{s}}$ occurred at the intermediate temperature of $60^{\circ} \mathrm{C}$ for the studied pressure.

Furthermore, temperature dependent rate coefficients accorded well with Arrhenius equation which states that the natural logarithm of rate coefficient is proportional to the inverse of absolute temperature (Connors, 1990).

However, we observed that the $k_{\mathrm{S}}$ value was barely influenced by pressure increased from 15 to $25 \mathrm{MPa}$ at $60{ }^{\circ} \mathrm{C}$, although the solubility was significantly improved. Previous reports have concluded that high extractability of BFRs in $\mathrm{ScCO}_{2}$ is generally achieved at high temperature and pressure (Gamse et al., 2000;

Suzuki et al., 2002).

Given consideration of both kinetic and thermodynamic aspects, an optimized operating condition $\left(60{ }^{\circ} \mathrm{C}\right.$, $25 \mathrm{MPa}, 1000 \mathrm{rpm}$ ) was also applied for the other four BFRs. As shown in Fig. 4, each BFR had a fingerprint spectrum within the band of $3800-2700 \mathrm{~cm}^{-1}$, resulting in a kinetic curve except for DBDBE, which does not contain any identifiable groups in its chemical structure. The specific spectral patterns were also verified by additional ATR-FTIR characterizations of pure BFR powders (Fig. S. 4). Actually, compared with ATR-FTIR spectra, the on-line FTIR spectra showed slight difference in a red shift phenomenon due to the formation of hydrogen bonding in $\mathrm{ScCO}_{2}$ and in relative absorbance intensity of different groups due to the existence of $\mathrm{ScCO}_{2}$ and sapphire windows (Fulton et al., 1993; Poliakoff et al., 1995). Regarding to the extraction performances of $\mathrm{HBCD}$, TBBPA-BDBPE, and TTBP-TAZ in $\mathrm{ScCO}_{2}$, saturated concentrations were lower but periods required to reach equilibria were all below one hour with comparison to TBBPA. DBDBE had the 

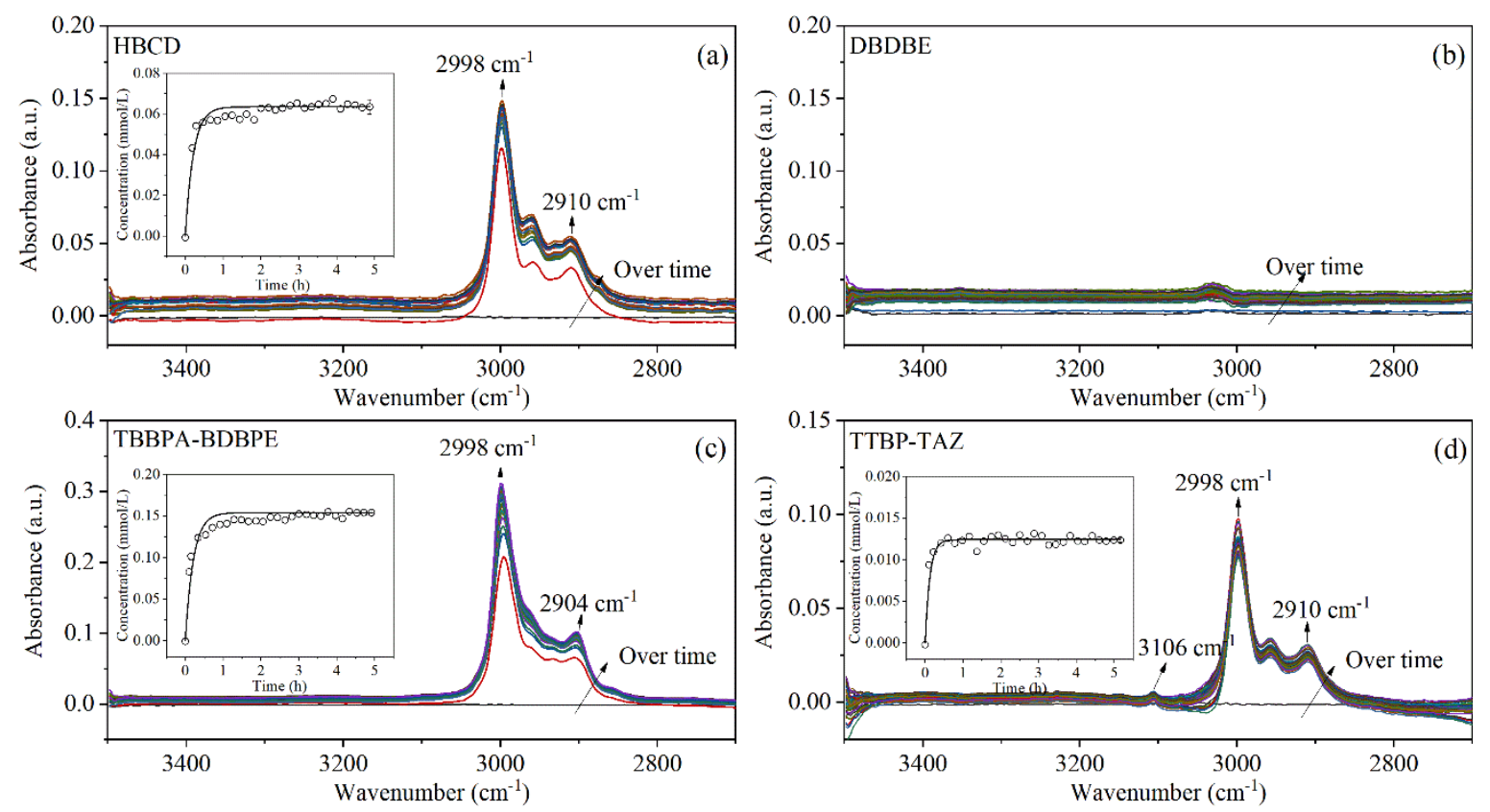

294

Fig. 4 FTIR spectra over extraction period and resulted kinetic curves of four BFRs at $60{ }^{\circ} \mathrm{C}, 25 \mathrm{MPa}$, and $1000 \mathrm{rpm}$.

\subsection{Effect of loading water as cosolvent}

In ternary systems, water was first included as cosolvent along with $\mathrm{BFR}$ and $\mathrm{ScCO}_{2}$ since a small amount of water added to $\mathrm{ScCO}_{2}$ was reported to increase the solubility of polar species in nonpolar solvent (Jackson et al., 1995). Other organic solvents were not included because of their high miscibility in $\mathrm{ScCO}_{2}$ inducing a large IR absorbance in the studied band. The FTIR spectra of TBBPA with loading of ultra-pure water $(200 \mu \mathrm{L}$, below saturation) were recorded over the textraction period (Fig. S. 5a). The absorbance caused by O-H stretching of $\mathrm{H}_{2} \mathrm{O}$ molecules did not interfere with the absorbance of TBBPA, thus kinetic curve for both TBBPA and $\mathrm{H}_{2} \mathrm{O}$ can be followed simultaneously (Fig. S. 5b). Water was faster to reach equilibrium than TBBPA. Despite an improved $c_{\mathrm{S}}$ of TBBPA by $7 \%$ in the presence of such small amount of water, its $k_{\mathrm{S}}$ decreased by $43 \%$ probably 


\begin{tabular}{|c|c|c|c|c|c|c|}
\hline \multirow[t]{2}{*}{ BFRs } & Cosolvent or matrix effect & $c_{\mathrm{s}}$ & $k_{\mathrm{S}}$ & $k_{\mathrm{D}}$ & $D_{\mathrm{e}}$ & $A A R D$ \\
\hline & & $\mathrm{mmol} / \mathrm{L}$ & $\mathrm{h}^{-1}$ & $\mathrm{~h}^{-1}$ & $\mathrm{~m}^{2} / \mathrm{h}$ & $\%$ \\
\hline
\end{tabular}

\section{$\mathrm{BFR}+\mathrm{H}_{2} \mathrm{O}+\mathrm{ScCO}_{2}$}

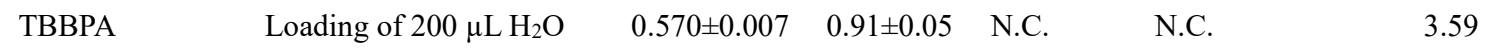

\section{$\mathrm{BFR} / \mathrm{ABS}+\mathrm{ScCO}_{2}$}

$\begin{array}{lcccccc}\text { TBBPA } & D_{\mathrm{ABS}}<0.25 \mathrm{~mm} & 0.333 \pm 0.001 & 1.78 \pm 0.08 & 1.08 \pm 0.05 & <1.7 \times 10^{-9} & 2.04 \\ & & & & & & \\ \text { TBBPA } & D_{\mathrm{ABS}} 0.25-1 \mathrm{~mm} & 0.331 \pm 0.001 & 0.49 \pm 0.08 & 0.25 \pm 0.02 & 4.0 \times 10^{-10}-6.3 \times 10^{-9} & 1.95 \\ & & & & & & \\ \text { TBBPA-BDBPE } & D_{\mathrm{ABS}}<0.25 \mathrm{~mm} & 0.219 \pm 0.004 & 1.94 \pm 0.37 & 1.32 \pm 0.08 & <2.1 \times 10^{-9} & 2.88\end{array}$

\subsection{Effect of ABS matrix}

To discover the matrix effect on extraction performance, BFR-free ABS resin was used as polymeric substrate to incorporate with BFRs, including TBBPA and TBBPA-BDBPE. The FTIR spectra of each extraction experiment are presented in Fig. S. 6. The extraction of ABS resin itself can also be observed, indicating that $\mathrm{ScCO}_{2}$ has a certain solvation power on $\mathrm{ABS}$, which can increase with temperature and pressure, leading to undesired dissolution of polymers or oligomers into $\mathrm{ScCO}_{2}$ (Sikorski, 1993; Zhao et al., 2018). In the case of TBBPA-incorporated samples, the effect of particle size was investigated on their extraction performance, as shown in Fig. S. 7. Obviously, the finer particle size, the faster the extraction process. The kinetic curve can be 
decomposed in two phases, with a first rapid extraction followed by a slower one. Their $c_{\mathrm{s}}$ of TBBPA were of similar value, $0.33 \mathrm{mmol} / \mathrm{L}$, lower than that of extraction without $\mathrm{ABS}$ matrix $(0.53 \mathrm{mmol} / \mathrm{L})$ due to the competitive dissolution of ABS polymers.

According to the broken and intact cell concept, analytes can be distributed at the outer surface of particles and embedded into their inner structure, just as BFR-incorporated ABS powders after grinding, resulting in easily accessible BFRs and less accessible BFRs, respectively (Sovová, 1994). Hence, the first rapid extraction of so-called washing process was controlled by solubilization in the well stirred reactor and $k_{1}$ can be assigned to $k_{\mathrm{S}}$ (Table 2). Furthermore, the sudden reduction of extraction rate after the first part indicates the completion of accessible BFR's extraction and the intra-particle diffusion resistance came into the effect (Srinivasan et al., 1990; Alexandrou et al., 1992; Grosso et al., 2010). Hereby, $k_{\mathrm{D}}$ can be calculated by $k_{\mathrm{D}}=k_{1}-k_{2}$ and $D_{\mathrm{e}}$ range can also be estimated according to Eq. (9). The TBBPA-BDBPE incorporated samples also supported the two-stage extraction process and the applied models provided very good approximation of experimental data with $A A R D$ lower than $2.9 \%$.

\section{Conclusions}

In this study, a supercritical reactor was assembled with on-line spectroscopic components to study $\mathrm{ScCO}_{2}$ extraction of BFRs. A notable feature of the apparatus, applicable beyond the current study, is the compatibility with both UV-vis and FTIR spectrometer using the same vessel, enabling to acquire more abundant spectral information of multi-component mixtures in real time and follow their kinetics. For quantitatively characterizing BFRs in $\mathrm{ScCO}_{2}$, FTIR spectroscopy is preferable to UV-vis due to its proper detection range and clearer spectral interpretation despite its limited spectra window. Five BFR molecules were studied using the on-line FTIR technique along with mathematical modelling. Stirring speed, temperature, and particle size were found to 
significantly influence extraction kinetics while pressure did not in the considered range. Sufficiently fast

stirring could eliminate limitations due to diffusion of molecules in $\mathrm{ScCO}_{2}$, and both higher temperature and

342 finer grinding size accelerate extraction kinetic. Therefore, high extraction rates could compensate for low

\section{CRediT authorship contribution statement}

Dong Xia: Methodology, Investigation, Formal analysis, Writing - original draft. Ange Maurice: Software.

Antoine Leybros: Methodology, Writing - review \& editing. Jong-Min Lee: Funding acquisition, Resources.

Agnes Grandjean: Funding acquisition, Formal analysis, Writing - Review \& Editing. Jean-Christophe P.

Gabriel: Funding acquisition, Resources, Conceptualization, Methodology, Writing - review \& editing,

\section{Acknowledgments}

We acknowledge intern Li-Yi Ang for her assistance in experimental investigation during her Final Year Project in Nanyang Technological University. JCG acknowledge the Region Pays de la Loire for "Young Researcher" funding for the purchase of Parr $\mathrm{ScCO}_{2}$ core reactor (1999). All authors acknowledge financial support from

SCARCE laboratory. SCARCE is supported by the National Research Foundation, Prime Minister's Office, 
Bartle, K.D., Clifford, A.A., Hawthorne, S.B., Langenfeld, J.J., Miller, D.J., Robinson, R., 1990. A model for dynamic

Bergman, Å., Rydén, A., Law, R.J., de Boer, J., Covaci, A., Alaee, M., Birnbaum, L., Petreas, M., Rose, M., Sakai, S., 2012. 
Carrott, M., Wai, C., 1998. UV- visible spectroscopic measurement of solubilities in supercritical CO2 using high-pressure

Covaci, A., Harrad, S., Abdallah, M.A., Ali, N., Law, R.J., Herzke, D., de Wit, C.A., 2011. Novel brominated flame retardants:

a review of their analysis, environmental fate and behaviour. Environ. Int. 37, 532-556.

Crank, J., 1979. The mathematics of diffusion. Oxford university press.

Fulton, J.L., Yee, G.G., Smith, R.D., 1993. Hydrogen Bonding of Simple Alcohols in Supercritical Fluids: An FTIR Study. 
Hojnik, M., Škerget, M., Knez, Ž., 2008. Extraction of lutein from Marigold flower petals-Experimental kinetics and modelling. LWT-Food SCI. Technol 41. https://doi.org/10.1016/j.lwt.2007.11.017. https://doi.org/10.1016/j.chroma.2012.04.032.

IUPAC, 1997. Compendium of chemical terminology. Blackwell Scientific Publications Oxford. https://doi.org/10.1351/goldbook.B00626.

Jackson, K., Bowman, L.E., Fulton, J.L., 1995. Water solubility measurements in supercritical fluids and high-pressure liquids using near-infrared spectroscopy. Anal. Chem. 67, 2368-2372. https://doi.org/10.1021/ac00110a007. of total polyphenols from soybeans. Czech Journal of Food Sciences 28, 206-212. https://doi.org/10.17221/200/2009-CJFS.

polystyrene: Quantum yields, products and influencing factors. Chemosphere 211, 943-951. https://doi.org/10.1016/j.chemosphere.2018.07.147.

Kim, Y.R., Harden, F.A., Toms, L.-M.L., Norman, R.E., 2014. Health consequences of exposure to brominated flame retardants: 
427

Microfluidics. Anal. Chem. 90, 4445-4451. https://doi.org/10.1021/acs.analchem.7b04425.

Laintz, K.E., Wai, C.M., Yonker, C.R., Smith, R.D., 1991. Solubility of fluorinated metal diethyldithiocarbamates in Supercritical carbon dioxide. J. Supercrit. Fluids 4, 194-198. https://doi.org/10.1016/0896-8446(91)90008-T.

Liu, K., Li, J., Yan, S., Zhang, W., Li, Y., Han, D., 2016. A review of status of tetrabromobisphenol A (TBBPA) in China. Chemosphere 148, 8-20. https://doi.org/10.1016/j.chemosphere.2016.01.023.

Lu, J., Han, B., Yan, H., 1999. UV-Vis spectroscopic studies of solute-solvent and solute-cosolvent interactions in supercritical carbon dioxide. Phys. Chem. Chem. Phys. 1, 3269-3276. https://doi.org/10.1039/A901854I.

Ma, C., Yu, J., Wang, B., Song, Z., Xiang, J., Hu, S., Su, S., Sun, L., 2016. Chemical recycling of brominated flame retarded plastics from e-waste for clean fuels production: a review. Renewable and Sustainable Energy Reviews 61, 433-450. https://doi.org/10.1016/j.rser.2016.04.020.

Madras, G., Thibaud, C., Erkey, C., Akgerman, A., 1994. Modeling of supercritical extraction of organics from solid matrices. AlChE J. 40, 777-785. https://doi.org/10.1002/aic.690400505.

Marioth, E., Bunte, G., Hardle, T., 1996. Supercritical fluid extraction of ABS-composites in order to separate organic flame retardants. Polymer Recycling(UK) 2, 303-308.

Maurice, A., Theisen, J., Gabriel, J.-C.P., 2020. Microfluidic Lab-on-Chip Advances for Liquid-Liquid Extraction Process Studies. Curr. Opin. Colloid Interface Sci. https://doi.org/10.1016/j.cocis.2020.03.001.

Nakajima, K., Kawakami, T., Ueno, T., Onishi, H., 2002. Method for treating thermoplastic resin composition containing flame retardant. U. S. Patent, United States.

Ni, M., Xiao, H., Chi, Y., Yan, J., Buekens, A., Jin, Y., Lu, S., 2012. Combustion and inorganic bromine emission of waste printed circuit boards in a high temperature furnace. Waste Manage. 32, 568-574. https://doi.org/10.1016/j.wasman.2011.10.016.

Nimet, G., Da Silva, E.A., Palú, F., Dariva, C., dos Santos Freitas, L., Neto, A.M., Cardozo Filho, L., 2011. Extraction of 
Poliakoff, M., Howdle, S.M., Kazarian, S.G., 1995. Vibrational spectroscopy in supercritical fluids: from analysis and

Sodeifian, G., Ghorbandoost, S., Sajadian, S.A., Ardestani, N.S., 2016. Extraction of oil from Pistacia khinjuk using 
471

472

473

Subra, P., Castellani, S., Jestin, P., Aoufi, A., 1998. Extraction of $\beta$-carotene with supercritical fluids: experiments and modelling. J. Supecrit. Fluids 12, 261-269. https://doi.org/10.1016/S0896-8446(98)00085-0.

Sunarso, J., Ismadji, S., 2009. Decontamination of hazardous substances from solid matrices and liquids using supercritical fluids extraction: A review. J. Hazard. Mater. 161, 1-20. https://doi.org/10.1016/j.jhazmat.2008.03.069.

Suzuki, M., Nakajima, K., Onishi, H., 2002. Method for treating flame retardant resin composition. U. S. Patent, United States. Tan, C.S., Liou, D.C., 1989. Modeling of desorption at supercritical conditions. AlChE J. 35, 1029-1031. https://doi.org/10.1002/aic.690350616.

Taylor, L.T., 1996. Supercritical fluid extraction. Wiley New York.

Villanueva-Bermejo, D., Fornari, T., Calvo, M.V., Fontecha, J., Coelho, J.A., Filipe, R.M., Stateva, R.P., 2020. Application of a novel approach to modelling the supercritical extraction kinetics of oil from two sets of chia seeds. J. Ind. Eng. Chem. 82, 317-323. https://doi.org/10.1016/j.jiec.2019.10.029.

Wang, H., Hirahara, M., Goto, M., Hirose, T., 2004. Extraction of flame retardants from electronic printed circuit board by supercritical carbon dioxide. J. Supecrit. Fluids 29, 251-256. https://doi.org/10.1016/S0896-8446(03)00073-1.

Wang, Z., Zhou, Q., Guo, H., Yang, P., Lu, W., 2018. Determination of water solubility in supercritical CO2 from 313.15 to 473.15 K and from 10 to $50 \mathrm{MPa}$ by in-situ quantitative Raman spectroscopy. Fluid Phase Equilib. 476, 170-178. https://doi.org/10.1016/j.fluid.2018.08.006.

Xiong, P., Yan, X., Zhu, Q., Qu, G., Shi, J., Liao, C., Jiang, G., 2019. A review of environmental occurrence, fate, and toxicity of novel brominated flame retardants. Environ. Sci. Technol. 53, 13551-13569. https://doi.org/10.1021/acs.est.9b03159.

Yu, G., Bu, Q., Cao, Z., Du, X., Xia, J., Wu, M., Huang, J., 2016. Brominated flame retardants (BFRs): a review on environmental contamination in China. Chemosphere 150, 479-490. https://doi.org/10.1016/j.chemosphere.2015.12.034.

Zhang, C.C., Zhang, F.S., 2012. Removal of brominated flame retardant from electrical and electronic waste plastic by solvothermal technique. J. Hazard. Mater. 221, 193-198. https://doi.org/10.1016/j.jhazmat.2012.04.033. 

707-720. https://doi.org/10.1016/j.chemosphere.2018.06.095.

495 Zhou, X., Guo, J., Lin, K., Huang, K., Deng, J., 2013. Leaching characteristics of heavy metals and brominated flame retardants from waste printed circuit boards. J. Hazard. Mater. 246-247, 96-102. https://doi.org/10.1016/j.jhazmat.2012.11.065.

497 Zuiderveen, E., Slootweg, J.C., de Boer, J., 2020. Novel brominated flame retardants-A review of their occurrence in indoor air, dust, consumer goods and food. Chemosphere, 126816. https://doi.org/10.1016/j.chemosphere.2020.126816.

499

500 